\title{
Aspects de la genèse de L'Escalier bleu
}

Le dossier génétique du recueil L'Escalier bleu procuré par le fonds Henry Bauchau de l'Université catholique de Louvain-la-Neuve compte près de 220 feuillets. Il comprend de nombreux feuillets manuscrits portant mention de la main d'Henry Bauchau «en travail » ou « une des premières versions", dont une grande partie concerne le seul poème «L'escalier bleu»; presque autant de feuillets tapuscrits; et quelques photocopies d'édition finale. Si nous avons de nombreux documents pour le poème «L'escalier bleu » et la partie intitulée La maison du temps, la partie Liant déliant y est pauvre, comportant seulement trois pages tapuscrites et 5 pages photocopiées de la première version publiée, mais aucun manuscrit.

L'écriture de l'ensemble du recueil s'est échelonnée entre 1958 (d'après les dires du poète ${ }^{1}$ ), et la fin de l'année 1963 ou le tout début de 1964. Au 15 décembre 1963, le poète relit le manuscrit de son recueil de poèmes et déçu, veut alors les retravailler; et au 21 avril 1964, il annonce ravi : «Finalement c'est le titre L'Escalier bleu qui est retenu par Gallimard pour mon recueil de poèmes. $»^{2}$; le recueil paraitra au mois de mai 1964. Son écriture a en gros suivi cette chronologie, avec toutefois des ajouts et une recomposition finale qui en brisent la linéarité, et approfondissent le projet, notamment dans le miroir d'enfance que constitue la section $L a$ maison du temps.

Le poème «L'escalier bleu », et certains poèmes de La maison du temps comme «La Glycine», "La Calèche» ont été exécutés parmi les premiers, vers 1958-1960, mais plusieurs poèmes de ce début du recueil

\footnotetext{
${ }^{1}$ Les dates «1958-1963» figurent sur la page de titre dans l'édition Actes Sud de 1986, non dans celle de 1964 chez Gallimard; elles sont également reprises dans l'édition Labor de 1995 et Actes Sud de 2009. Elles se trouvent également manuscrites, ainsi que la dédicace "à Jean Amrouche» sur un feuillet non numéroté du dossier du fonds Bauchau, le titre étant lui dactylographié en gros caractères, l'adjectif «bleu » disposé de manière légèrement décalé (en marche d'escalier ?) après "L'escalier». D'autre part deux tapuscrits du poème "L'escalier bleu » portent sur leur dernier feuillet numéroté l'un A112, l'autre A132 la mention finale : « 5-26 janvier 59 ».

${ }^{2}$ GM, p. 352 ; voir aussi pp. 274, 292 et 326.
} 
ne seront créés que tout à la fin, au cours de l'année 1963 ; en particulier, les poèmes «Le Forestier» et «La volière ouverte » sont sans doute parmi les derniers à être écrits à la fin de 1963 et viennent étoffer la partie plus nettement biographique placée en tête du recueil ${ }^{3}$. Les dates précises de nombreux poèmes de ce début du recueil échappent pour l'instant à notre connaissance puisque le Fonds Bauchau ne dispose pas des journaux inédits des années 1958-1960 . Mais le dépouillement des manuscrits poétiques fait apparaître, par la densité de travail qu'ils révèlent, que $L a$ maison $d u$ temps et le poème liminaire éponyme «L'escalier bleu» constituent des moments intenses du déploiement de l'écriture d'Henry Bauchau, donc de sa libération psychique par l'écriture ${ }^{5}$, et que, au-delà de «L'escalier bleu » qu'il relit souvent et avec beaucoup d'émotion ${ }^{6}$, des poèmes comme «Les matières», «Les chambres », "Les chevaux morts », "La Glycine », et les deux derniers écrits, "Le Forestier» et "La volière ouverte», ont beaucoup compté dans son cheminement poétique et personnel.

D'une certaine façon, à ce jour, la genèse du recueil L'Escalier bleu commence et s'achève sur des lignes de fuite : pas de date exacte, pas de témoignage de journal pour le début, qui de toute façon plonge aux territoires les plus enfouis des souvenirs de l'enfant, voire dans une «mémoire immémorielle »: le souvenir de la pierre bleue de l'escalier est aussi relié à la «Pierre de l'homme originel, père confus / homme encor englouti dans la mer, homme d'algues /et de sel ingénu $»^{7}$, comme le dit le poème. Il est probable qu'Henry Bauchau a porté très tôt en lui, de manière confuse, les vrilles de l'escalier bleu et la représentation de la maison de Blémont comme lieu de sensualité et d'éveil à l'existence, puis

\footnotetext{
${ }^{3}$ L'écriture des poèmes de Liant déliant, dernière section au sommaire à partir de 1986, se situe en gros entre avril 1962 et mai-juin 1963 comme permet de le voir Journal de La Déchirure 1960-1965.

${ }^{4}$ Henry Bauchau a cependant bien tenu un journal dans ces années-là comme le montre sa réflexion : "relu mes journaux de 1959 à 1962. Ils donnent une vision bien incomplète de ma vie. » (GM, p. 382.)

${ }^{5}$ Voir en date du 13 juin 1960 : «Pour moi en écrivant, ce que je tente encore c'est de me réaliser. Je suis très en deçà de la position de Sartre. » (GM, p. 68.)

${ }^{6}$ Voir GM, p. 25 (31 janvier 1960), pp. $43-44$ (13 et 14 mars 1960), p. 183 (17 mars 1962 : «Je ne sais pas ce que j'ai fourré dans ce texte, mais je ne puis le relire sans avoir les larmes aux yeux. Et effectivement je l'ai écrit en pleurant, ce qui me demeure obscur »), p. 292 (25 mai 1963).

7 «L'escalier bleu », Esc, p. 66.
} 
de déclencheur de l'écriture, comme moment de son histoire reliée à une histoire mythique et quasi archétypale ${ }^{8}$. Mais le chercheur n'est guère mieux fixé sur la date finale de la genèse et composition de ce recueil, sauf à admettre, mais avec quel bien-fondé ?, que la dernière version publiée serait la version de référence. La publication du recueil est en effet marquée, entre 1964 et 2009, par plusieurs réécritures et recompositions qui lui donnent au fil du temps une structuration mouvante, comme dans un kaléidoscope, dans lequel des vers ou poèmes, entrant ou sortant, paramètrent différemment la configuration générale. De ce fait, le jeu des éditions successives entre dans l'ensemble du dossier génétique et il faut considérer les textes publiés puis modifiés comme des avant-textes des publications ultérieures. Ceci conduit à insister avec les généticiens sur la nécessaire circonspection dont il faut faire preuve face au sens et à l'interprétation possible du texte, sans cesse remis en question par ce que révèlent les manuscrits et les éditions successives : "La plupart des manuscrits apportent un démenti formel à la possibilité de conclure sur le sens des textes, écrit Pierre-Marc de Biasi, [...] le document de genèse proroge l'inachèvement, l'installe au cœur du texte parachevé, transforme l'interprète en explorateur des possibles »? L'examen du dossier génétique de L'Escalier bleu étendu à ses diverses éditions confirme cette observation.

Cette incertitude sur l'extension à donner à la notion de genèse chez Henry Bauchau se double, comme chez d'autres poètes du XX $\mathrm{X}^{\mathrm{e}}$ siècle, de l'incertitude sur la notion de recueil dans son œuvre, sa mort venant interrompre, et non clore, une genèse sans fin et instituer pour un même titre des recueils proches et toutefois différents.

L'Escalier bleu, comme d'autres recueils d'Henry Bauchau, a connu en effet quatre éditions distinctes du vivant du poète ${ }^{10}:$ la première édition en recueil isolé chez Gallimard de 1964 ; l'édition dans le volume Poésie chez Actes Sud de 1986 ; l'édition dans Heureux les déliants, Poèmes 1950-

\footnotetext{
8 Voir la thèse de Corina Dambean, «Poétique du minéral dans l'œuvre d'Henry Bauchau », Université de Cergy-Pontoise, mai 2012, à paraître aux éditions Honoré Champion.

9 Pierre-Marc de Biasi, La Génétique des textes, Nathan Université, 2000, p. 86.

${ }^{10}$ Le poème «L'escalier bleu » a aussi connu une édition en revue à la NRF n 104 , en août 1961 (pp. 231-235). On ne note qu'une interversion de deux vers : "Quand l'eau sainte des mots, troublée, retombe en larmes / Ces rires mutilés s'écrasent sur les pierres ». Nous remercions Jérémy Lambert qui nous a donné ce renseignement.
} 
1995 aux éditions Labor en 1995; l'édition dans Poésie complète, de nouveau chez Actes Sud, en 2009.

Ces quatre éditions diffèrent toutes les unes des autres sous différents aspects. Le premier changement notable dès la seconde édition de 1986 est la densification du recueil pour des raisons éditoriales par la publication de plusieurs poèmes par page là où l'édition de 1964 consacrait une page à chaque pièce, assurant une plus grande résonance aux poèmes et une lecture plus lente.

Le second changement porte sur la structuration du recueil qui se simplifie en passant de cinq sections dans la première édition (poème liminaire inclus) à trois sections. Il porte également sur le paratexte qu'Henry Bauchau a changé d'une édition à l'autre ${ }^{11}$. Il y a là tout un travail très fin de mise en cuvre et d'advenue du recueil dans ses lignes de force, qui montre la très lente maturation dans l'esprit du poète de la vision d'ensemble du recueil, une décantation, qui le fait parvenir à un recueil allégé.

Plus significatif encore, on note la suppression de plusieurs poèmes entre 1964 et 1986 : si «L'harmonica » et «L'oubli vert» se sont fondus respectivement dans «Douze regards sur une enfance» et dans «Oubliant», cinq poèmes ont été supprimés lors de la première réédition, et deux autres, non des moindres puisqu'il s'agit de « La calèche » et « Nous ne sommes pas séparés », disparaîtront dans l'édition ultime de 2009. «La calèche » s'est définitivement perdue « sur la montée du temps » ${ }^{12}$. Le poème "Nous ne sommes pas séparés» donnera son titre au recueil publié en 2006 qui accueillera aussi le poème retranché de L'Escalier bleu, avec un changement notable puisque "nous ne sommes pas séparés de la mort» deviendra en 2006 et 2009 «Nous ne sommes

11 À partir de 1986, les dates 1958-1963 sont systématiquement ajoutées à la suite du titre. La dédicace «À Ariane » du poème «Douze regards sur une enfance » est précisée en «à Ariane Mnouchkine» en 1986, et les astérisques séparant les strophes disparaissent en 1995. «Pleurant ne pleurant plus » perd son exergue dès 1986 ("That I have folowed beanty reward to know there's a God for fish», Gregory Corso). Le vers antépénultième «demande à Gregory» est aussi supprimé, mais seulement dans l'édition de 2009). Des titres sont modifiés : "La fenêtre d'images » sert de titre à une troisième section en 1964, section qui disparaitra dans la nouvelle structuration, et le poème appelé «La fenêtre d'images » dans l'édition de 1986 s'appelait « Mérence » dans celle de 1964. «L'oubli vert» de 1964 s'intitule « Oubliant » en 1986.

12 «Les chevaux morts », Esc, p. 75. 
pas séparés de la Terre » et «nous ne sommes pas séparés de la vie ». Le déplacement externe de ce poème, publié dans le recueil qui suit la mort de Laure ${ }^{13}$, et ces variantes lexicales notables qui situent le poème du côté de la mort plus que du côté du vivant- induisent alors une tout autre lecture du poème; cependant, même dans cette nouvelle position, il entretient des liens latents avec l'environnement de sa première publication et avec sa genèse plus lointaine ${ }^{14}$. Nous parlerons ici d'une construction poétique en stolon, pour filer une première métaphore sur le processus génétique chez Henry Bauchau et il nous semblerait intéressant que les chercheurs se penchent sur la circulation interne des poèmes à l'intérieur de l'œuvre, sur ce maillage ou cet agrafage des recueils entre eux, qui assure des connexions arachnéennes et une continuité de l'œuvre œuvrant à rebours du temps : le recueil antérieur continue de fermenter par ces poèmes-témoins dans le recueil ultérieur, et celui-ci reste relié par un cordon visible à l'œuvre passée ${ }^{15}$.

Autre changement: le poète travaille au déplacement interne de poèmes entre la première (1964) et la seconde (1986) édition du recueil L'Escalier bleu. C'est dans la section La Maison du temps que ces déplacements sont les plus nombreux. La mise en concordance des sommaires des quatre éditions (1986 et 1995 ayant des sommaires identiques) montre les entrecroisements nourris auxquels a procédé le poète, rendant délicate, sinon impossible, la circonscription du recueil comme un ensemble clos et signifiant. Pour aller plus loin, le critique doit se livrer à l'étude très fine des exigences qui ont guidé les choix du poète dans l'ordonnancement de chaque section, mais devant la multitude des hypothèses possibles, risque de rester dans l'aporie. Cela revient à accepter une fluidité de l'œuvre comme échappant à toute structuration définitive et à la lire comme une œuvre relative, transitoire,

\footnotetext{
13 Qui rappelle les changements de date de certains poèmes répartis entre "Autrefois » et «Aujourd'hui » dans Les Contemplations (1856) après la mort de Léopoldine (1843).

${ }^{14} \mathrm{Ni}$ l'édition en recueil chez Actes Sud en 2006, ni la reprise dans Poésie complète en 2009 ne précise les dates de composition des poèmes de Nous ne sommes pas séparés. Dans l'édition de 2009 toutefois, le recueil est pris entre des recueils datés 1987-1995 (Heureux les déliants) et Petite suite au 11 septembre 2001 ou les poèmes inédits de L'Accueil datés de 2006-2009. L'organisation chronologique des recueils induit dans l'esprit du lecteur la suggestion d'une genèse lui correspondant.

15 Jérémy Lambert nous a également fait savoir que le poème "L'Harmonica » commence d'être travaillé parmi les poèmes de Géologie.
} 
passagère, ce qui s'accorde avec le propos de Déluge. Ceci engage aussi la relation au temps dans la création. Ce qui est certain, c'est que le travail sur la notion de «recueil» chez Henry Bauchau reste à faire et sera certainement très productif.

On observe enfin, entre les différentes éditions, de nombreuses variantes d'écriture d'un poème à l'autre, et ce jusque dans la dernière édition de 2009. Il s'agit parfois de modifications infimes, comme des changements de ponctuation ${ }^{16}$, ou de variantes lexicales; parfois une strophe est supprimée ou déplacée d'un poème à l'autre. Un des exemples les plus marquants de réécriture est le poème «Pleurant ne pleurant plus », qui subit une réduction importante au cours de ses trois rééditions, passant de 57 vers (1964) à 39 vers (1986), puis 35 vers (1995) pour finir à 24 vers en 2009, soit moins de la moitié du poème initial. Or ce poème est les quatre fois dédié «En mémoire de Jean Amrouche », et Jean Amrouche est aussi les quatre fois dédicataire de la totalité du recueil. Le travail de suppression et de contraction le plus important s'effectue entre les deux premières versions (1964 et 1986). Cette réécriture fait ressortir la sûreté de jugement acquise par le poète sur son propre travail : il supprime en effet des vers de moins bonne facture du début du poème ${ }^{17}$; mais il les supprime d'autant plus facilement que ces vers étaient empreints d'une violence contenue et portaient trace autant de l'émotion ressentie à la mort de son ami que des violences de l'époque. Bauchau décontextualise, deshistoricise son poème: il fait disparaittre l'allusion aux casbahs dans la troisième version, puis l'allusion aux Algériens en liesse de la fin de la guerre d'Algérie dans la quatrième version. Le poème dans sa version «finale» est beaucoup moins circonstancié, plus ouvert à l'universel et plus intériorisé ; il apparaît comme proche de la rhétorique des poèmes plus tardifs de Nous ne sommes pas séparés ou de L'Accueil qui date des années 2006-2009, c'est-àdire du moment où le poète prépare aussi la réédition de Poésie complète chez Actes Sud et pour ce faire, révise ses productions antérieures. Le

\footnotetext{
${ }^{16}$ L'une des plus incertaines est sans doute celle qui porte sur la fin du vers de «L'escalier bleu » qui apparaît en 1964 : « Mérence me restait, mais laquelle tombée...» (p. 15), en 1986 et 1995 : "Mérence me restait, mais laquelle tombée. » (p. 63 et p. 242) et en 2009 : «Mérence me restait, mais laquelle ? (p. 68.)

${ }^{17}$ «Je suis revenu à la cité d'avril / Elle m'attendait avec son air de fille sauvage / elle me regardait avec ses yeux de fille méchante / et m'a mesuré avec son couteau / Douleur j’allais les mains ouvertes / Les portes n'étaient plus fermées », Esc, p. 59.
} 
regard rétrospectif sur le poème conduit à cet élagage et mise à distance des crises de l'actualité devenue histoire, nuançant alors le titre du poème d'un sens autre: "Pleurant ne pleurant plus». À travers ces suppressions, un travail de deuil s'est accompli par rapport au dédicataire Jean Amrouche, le poème «abouti » de la dernière édition rayonne d'une sérénité mieux assumée et devient un hommage apaisé. Suivre l'évolution d'écriture d'un poème comme celui-ci donne en échantillon un aperçu de l'évolution de la poétique d'Henry Bauchau, passé de poèmes peut-être plus narratifs ou plus attachés aux circonstances et empreints d'inquiétude, à une écriture plus épurée et allusive. Il serait intéressant de voir comment l'affirmation de la poétique de Bauchau accompagne aussi les mutations poétiques de son temps. Le Journal de La Déchirure témoigne de ses tiraillements entre des poétiques totalement opposées : d'un côté Hugo: «Il est certain que Hugo se rapproche » note-t-il le 25 août 1961 ${ }^{18}$; Claudel et les Cinq grandes Odes ${ }^{19}$; Saint-John Perse dont il reconnait l'influence et dont il défend Amers contre l'avis de Jouve ${ }^{20}$; de l'autre Philippe Jaccottet dont il admire le «style plein, concret, modeste »: "Combien plus sûr son instrument verbal que celui dont je dispose et souvent abuse $»^{21}$. Les manuscrits font apparaittre une véritable tension entre ces deux formes de poétiques. L'empreinte persienne est très nette dans une certaine rhétorique et rythmique qui apparaissent dans certains passages que le poète gommera en cours de travail, comme au feuillet A50 de «L'escalier bleu » : «l'homme encore englouti dans la mer, homme d'algues / et de sel vagabond, ingénu mais le seul / à entendre le temps plus vaste des racines / aux mouvements des grandes pierres immobiles » (notons les deux alexandrins). Certains passages des manuscrits de «La calèche » retentissent aussi d'accents apollinariens, de l'Apollinaire de «Cortège » ou de «La maison des morts » :

\footnotetext{
18 GM, p. 152.

19 "La fin de l'ode "La Muse qui est la Grâce" me paraît un des sommets de sa poésie et j’ai lu peu de choses aussi courageuses et aussi dures. », Ibid., p. 334.

${ }^{20}$ Ibid., pp. 81-82, 243 et p. 175 : «Quelles trouvailles qui ne sont pas seulement de langage mais de rythmes, des élans, des perceptions les plus fines ou les plus altières. Plus que sous celui de l'exil, c'est sous le signe de l'embrasement et de la louange que se trouve son œuvre. Et qu'un mouvement n'aille pas sans quelque inflation, c'est naturel, l'océan aussi se répète. »
}

${ }^{21}$ Ibid., pp. 328-329. 
La beauté ne savait qu'un mot, que j'ai perdu.

Je le cherche toujours sachant bien qu'on ne trouve

qu'après de longs/grands/longs efforts mais sans raison. J'ai pris

le łong/lent chemin des mots, la phrase où je me trouve

égaré, mais l'enfant qui me tient par la main

je sais que s'il revient un jour dans la remise

s'il joue dans les buissons et voit quelque merveille

il voudra me le dire et qu'alors il se peut

sorti de la maison du temps que j'y retourne (A177)

Le 25 mars 1960, Henry Bauchau écrit dans son journal: "Me souvenir du profit que j'ai eu pour "Les Remises" et "L'escalier bleu" à faire un brouillon en prose» (GM, p. 49). Pourtant ce qui frappe lorsqu'on aborde les feuillets manuscrits du recueil "L'escalier bleu », c'est que le rythme poétique apparait d'emblée, et qu'il est moulé sur un dodécasyllabe : c'est le mètre dominant dans presque tous les poèmes manuscrits, ce qui ou bien laisse supposer qu'il a existé d'autres manuscrits avant ceux dont nous disposons, ou bien que le rythme intérieur dont parle le poète à propos de l'émergence du poème, du moins au stade de l'écriture de "L'Escalier bleu» et La maison du temps, est déjà dirigé par un rythme dodécasyllabique fortement intériorisé. Seul le manuscrit de «La remise » (qui deviendra «La Glycine ») semble d'un rythme plus discontinu et prosaïque. Le poète semble travailler par masses relatives, d'abord une page et demie à deux pages, correspondant aux différentes parties différenciées par des blancs dans le poème publié. Puis il recopie et capitalise progressivement le poème dans sa longueur. Les brouillons sont raturés, mais somme toute assez peu, et ils restent presque toujours lisibles; certains piétinent, recopient plusieurs fois la même phrase ; mais s'il s'agit réellement de premiers jets, ils sont déjà consistants, rythmés, et semblent aboutir plus vite que ne le laisse à penser le journal. L'écriture première paraît abondante, projetant en mots une vision déjà mûre. ${ }^{22}$

Si les premières versions proposées sont loin de la version publiée, divers fragments en sont très proches, comme le poème "La canicule » issu tout droit des premières lignes manuscrites de «L'escalier bleu » : les feuillets A 42 et A 43 proposent ainsi :

${ }^{22}$ Voir 5 décembre 1963 : «Le réel se colore par l'image et par le sens, à l'état brut il est peut-être insupportable d'opacité. » (GM, pp. 322-323.) 
Un jour je saisirai la belle, la rebelle

ta connaissant par sa crinière vé

et connaissant

par sa crinière végétale eonnaissant,

je vaincrai, je serai vaincu dans la merveille

blonde par un vrai ciel, mouvante, violette.

Et l'offrirai

Je l'offrirai, o terre arable, corps de trèfle

Au monde en libation pour un regard brûlé

Et pour vos cris, coquelicots, en sacrifice

Ce qui devient dans la première édition de 1964 :

Un jour je saisirai la belle, la rebelle

Par sa crinière végétale

Un jour je t'offrirai, arable corps de trèfle

au ciel en libation pour un regard brûlé

et pour vos cris, coquelicots, en sacrifice. ${ }^{23}$

Certains vers signalent leur importance par l'acharnement avec lequel le poète y revient et les travaille. Pressentant parfois l'adéquation d'une image avec l'intuition intérieure et la trouvaille lexicale heureuse, il se saisit d'une formule et la réécrit de multiples fois dans divers contextes jusqu'à trouver forme et emplacement satisfaisants. Ainsi en est-il du syntagme "la terre sans semelles» qui figure à la fin de la première strophe de «L'escalier bleu » avec une quasi hypallage suggérant l'accord de l'enfant avec le monde: "et l'enfant qui touchait la terre sans semelles $»^{24}$.

Ce vers apparait dans le premier feuillet (A42) sous la forme «Et septembre sentait la terre sans semelles $»^{25}$ où l'hypallage est avérée, puis, «l'enfance où je touchais la terre sans semelles » (A69), «L'époque où je touchais la terre sans semelles » (A76), «l'enfance encor mouillée de terre sans semelles» (A79), et si le feuillet A133 propose la forme

\footnotetext{
23 «La canicule», Esc, p. 48. Le poème ne subit pas de modifications dans les éditions ultérieures. Voir aussi au 22 mars 1960: "J'ai travaillé dimanche presque toute la journée à ce petit poème extrait de L'escalier bleu: "Un jour je saisirai la belle, la rebelle..."» (GM, p. 47.)

${ }^{24}$ Ibid. p. 11 ; édition Actes Sud 2009, p. 65.

${ }^{25}$ Cette même version apparait sur le feuillet A82, sur les tapuscrits A94, A102, A113.
} 
«définitive », elle est encore retravaillée par la suite en «Septembre découvrait la terre sans semelles» (A139) ${ }^{26}$.

Il en est de même pour trois vers qui seront placés dans l'édition de 1964 au final de "Les chevaux morts", mais qui commencent d'apparaître dans le cours du travail sur le poème "La calèche ». Le passage visiblement cherche à exprimer sur un ton nostalgique le basculement de l'enfance dans l'adolescence à partir de modèles animaux :

Tandis que tout autour grandissaient les poussins

$[\ldots]$

Tout se mit à finir très vite à s'agrandir

Un poulain gandissait sur des genoux étroits

Et tout se mit à s'agrandir d'adolescence ${ }^{27}$

Ces trois vers apparaissent dans l'état où ils paraitront sur le feuillet A184 :

Sur leurs genoux étroits, les poulains grandissaient

c'était la fin d'un temps devenu très ancien, trop vite, un temps naif sur des pavés pointus. ${ }^{28}$

Mais Henry Bauchau retravaille ce fragment de manière obstinée, en proposant d'affilée six autres versions avec notamment une formule très persienne qui ressurgira dans les versions ultérieures :

Je vous parle d'un temps devenu très ancien

trop vite, un temps naïf sur des pavés pointus ${ }^{29}$

Comme un musicien qui joue et rejoue le même passage d'une partition jusqu'à obtenir la fluidité voulue, Bauchau s'acharne sur ce court passage qui a un retentissement particulier dans la vision sensible et symbolique de son passé et sur cette représentation de la fragilité de l'enfance. Ce fragment en travail réapparaît encore un grand nombre de

\footnotetext{
26 Un feuillet tapuscrit non numéroté entre les feuillets A139 et A140 reproduit plusieurs fois la formule.

${ }^{27}$ FHB A179. ; repris avec variante «s'engourdir » (A181).

${ }^{28}$ FHB A184 et édition de 1964, p. 28. Les deux premières virgules ne figurent pas sur la version manuscrite. Voir aussi feuillet A183.

${ }^{29}$ FHB A184. Cette même version réapparaît sur les feuillets A198, A204.
} 
fois, permutant entre les manuscrits de «La calèche », et ceux de «Les chevaux morts $»^{30}$ où ils prendront finalement place.

Cette hésitation sur la place à donner à tel ou tel fragment, nous paraît également caractéristique de ses tâtonnements, et s'observe à plus grande échelle, ce qui met à jour la matrice commune dont émergent les différentes écritures d'Henry Bauchau. Dire l'escalier bleu, site originel de l'histoire de sa personnalité, recouvre pour lui un enjeu (re)constructeur, ce qu'il dit dans le Journal de La Déchirure : «c'est bien le lieu où j'ai commencé à me perdre, un de ceux où je puis me retrouver $»^{31}$. De l'image de cet escalier bleu vont naître le poème, le recueil et le roman ${ }^{32}$. Plus qu'un travail en stolon, il s'agit cette fois d'une sorte de division cellulaire du texte, d'un déploiement de l'œuvre à partir d'une même représentation originelle de soi. Nous trouvons plusieurs exemples de ces écritures siamoises qui vont se disperser et se dilater en plusieurs textes. Les premières pages manuscrites du poème «L'escalier bleu » font mention de l'oncle Jules :

Là se trouvait souvent l'oncle Jule[s], homme noir

dont les guêtres sentaient la pulpe des automnes,

la moustache hérissée il dirigeait la ferme

et son tonneau anglais, ses juments pommelées

martelaient l'heure à nos pavés d'horloge claire. ${ }^{33}$

Il parlait en patois aux hommes dont l'effluve

disait quelle récolte ils faisaient, quels chevaux

ils menaient aux labours ou à la sucrerie

et dans quels chemins creux du soir, les pécheresses

celles qu'on regardait le dimanche à la messe

secrètes, sans regards, chanter au banc des filles. (A47)

Or la figure de l'oncle Jules est escamotée en cours du travail et disparaît du poème $e^{34}$, mais elle (ré)apparaît dans La Déchirure sous des syntagmes et des images proches de ceux du manuscrit du poème :

\footnotetext{
${ }^{30}$ FHB A 198, A201, A 204.

31 GM, pp. 47-48. La Déchirure le redit: «Je trouve l'escalier au départ de toutes mes sensations et de tout ce que j'aime » (p. 72).

32 Ce que l'hésitation sur les titres à attribuer confirme aussi.

${ }^{33} \mathrm{La}$ formule est là encore très proche de la poétique de Saint-John Perse.

34 «Il fallait traverser au milieu des fumées / L'office où s'affrontaient le charron et les gardes / dont les guêtres sentaient la pulpe de l'automne. / Plus lente était la voix des
} 
On trouvait souvent à l'office M. Jules qui se chauffait les mains au-dessus du poêle. Durant le petit-déjeuner, on entendait sonner sur les pavés, le bruit clair du trot de sa jument et des deux roues de son tonneau anglais. Il avait la peau cuivrée, de fortes moustaches noires et ses guêtres, en toute saison, sentaient la pulpe de betterave. [...] [il] continuait à parler patois avec le charron, les charretiers, les bouviers ou les gardes. (D, p. 103)

Le portrait du cocher Victor qui apparaît et dans le poème «Les Chevaux morts» et dans le chapitre «La chaleur violette» de La Déchirure montre là encore la proximité et l'interaction d'écriture entre plusieurs poèmes et le roman à partir de la même source mémorielle. Le feuillet A207 porte ceci :

Mais surtout il y avait la calèche [la vieille qu'on n'attelait plus, mais qu'on gardait pour

si jamais. Elle était noire avec il me semble des brancards verts et un fouet toujours bien astiqués les harnachements $[\ldots]$

Victor, dans sa jeunesse était monté [en postillon avec une veste rouge et un grand chapeau gris qui était accroché sur son lit, un lit [de paille au-dessus de l'écurie $[\ldots]^{35}$

On retrouve de ce passage trois vers du poème « La calèche » :

Le grand amour c'était la calèche $[\ldots]$

La calèche gardée encor pour si jamais

$[\ldots]$

Elle avait été noire et brillante

L'image des harnais réapparait dans le poème "Les chevaux morts » qui, cependant, est le moins proche de l'état manuscrit :

les harnais des chevaux défunts pendaient toujours

hommes de charrue / qui mènent labourer les juments dans la plaine / et dans les chemins creux les belles braconnières / pénitentes qu'on voit, le dimanche à la messe / sourdes et sans regards, chanter aux bancs des filles. ", «L'escalier bleu », loc. cit,. p. 12.

${ }^{35}$ FHB A207 ; « ils brillaient » de lecture douteuse. 
glorieusement polis et Victor le cocher

faisait là dans un trou plein d'ombre sa litière.

En revanche le même imaginaire et quelques éclats de la même représentation irriguent le texte du roman :

Au second palier s'ouvre un corridor, très obscur, sur lequel donne la chambre de Victor. [...] Il l'éclaire jour et nuit avec une lampe tempête qui projette de belles ombres au mur où l'on voit briller des mors, des gourmettes et des trophées équestres. [...] Au-dessus de son lit pend un tricorne à galons d'or qu'il a porté dans sa jeunesse, quand il montait en postillon. ( $D$, p. 113.)

Les textes s'engendrent d'une commune matrice, progressent en rhizomes à partir de bribes ou projections verbales. Une partition s'opère entre les différentes formes d'écriture, ce dont témoigne le Journal :

Le mouvement par lequel j'entame le roman et celui par lequel j'écris le poème sont le même. C'est le retour vers le passé pour le voir réalisé, reconstruit dans une vision nouvelle.

Le poème dit certaines choses qui ne peuvent être dites autrement, il le dit sur un mode qui n'est pas celui de la prose. Il ne faut pas mélanger les deux choses mais les mener parallèlement.

Au même moment j'ai envie de reprendre pour le roman le thème de «L'Escalier bleu ». (GM, p. 34.)

Bauchau écrit ceci le 26 février 1960, et il a commencé d'écrire depuis moins de dix jours La Déchirure, le 19 janvier 1960". Ceci confirme le propos de Geneviève Henrot dans Henry Bauchau poète, le vertige du seuil: «[...] la résille thématique qui enlace poèmes, romans, théâtre témoigne [...] plus souvent d'un processus de prosaïsation $[\ldots]$ que d'un processus de poétisation $[\ldots] »^{37}$.

Nous voudrions pour finir nous intéresser aux chutes des manuscrits : Bauchau a supprimé des pans entiers de certains poèmes en cours de

\footnotetext{
${ }^{36} \mathrm{D}, \mathrm{p} .281$.

${ }^{37}$ Geneviève Henrot, Henry Bauchau poète. Le Vertige du senil, Droz, 2003, p. 41.
} 
travail. Les manuscrits des deux poèmes non encore dissociés «La calèche » et «Les chevaux noirs » portent trace d'un double épisode qui disparaitra de la version finale. Ce passage combine tout à la fois nourriture, enfance et animalité sous l'angle d'une forte sensualité. Un geste de Victor est noté, Victor dont la présence dans le roman La Déchirure $^{38}$ est fortement marquée du signe de l'olfactif et qui est amateur d'avoine :

Toujours avant de la [l'avoine] verser dans les mangeoires

Il en prenait un peu dans sa main, la flairant

puis la faisait couler un temps sur son visage.

Il nous donnait des grains en cachette à manger (A178) ${ }^{39}$

Plus bas dans le même feuillet, Bauchau avait écrit :

Dans la calèche il y avait un sac de blé

pour les poussins, on pouvait dans la matinée

les nourrir et sentir dans nos mains

le minutieux baiser du bec

te baiser sec et avide, minutieux

le court baiser chatouilleux du bec (Ibid.)

Le poète reviendra de manière répétée sur cet épisode et sur la figure des poussins qui réapparaissent sur les feuillets A179, A181, A191, A202, et encore dans plusieurs feuillets de «La Glycine» (A206, A 208) avant de disparaitre. Tous ces fragments supprimés et le motif du poussin entraient dans une double isotopie des poèmes de La maison du temps, d'une part la sensualité, l'éveil à l'existence par le tactile et l'olfactif, l'apprentissage de la vie par les sens, la découverte de la matière ; d'autre part le sentiment de fragilité lié à l'enfance et à un sentiment de faiblesse inné. Dans le manuscrit de «La Glycine », les poussins couvés naissent, et le poète décrit ceci :

$[\ldots]$ puis les poussins venaient [d'abord humides

on voyait la peau sous le duvet j'avais pitié

parfois l'un venait plus tard, avec du sang/peine [je sentais

que c'était moi, souvent il mourait j’ai vécu

\footnotetext{
${ }^{38}$ D, p. 113.

${ }^{39}$ Le dernier vers est repris dans la version publiée de « Les chevaux morts ».
} 
car j’ai eu la colère

Ces couvaisons de poussins ont totalement disparu des poèmes de «L'escalier bleu », comme du roman La Déchirure. Il n'en subsiste qu'une discrète allusion à travers "les œufs frais du matin dans leurs coques légèrement brunies » et la métaphore filée entre l'œuf, la maison chaude, Mérence, et «l'obstacle innocent de la coque » qu'il faut ouvrir «à petits coups de cuillère » « pour éprouver nos forces et parvenir à la saveur ${ }^{40}$, comme si le motif de la faiblesse avait été retourné en force, en «force faible» selon le mot du philosophe François Laruelle ${ }^{41}$. Certainement, dans ce doublet des poulains et des poussins qui apparaissait dans le cours du travail, le poète a-t-il choisi à bon escient de tuer les poussins dans l'œuf et de garder le motif des poulains, souvenir possible d'Anabase?, gage de grandeur épique et de figures hérö̈ques ou militaires, motif aussi du souvenir de son éveil sexuel.

Mais «La calèche » aussi a sombré, puisque le poème sera supprimé dans l'édition de 2009, ce qu'un lecteur peut regretter. Plusieurs hypothèses peuvent être émises, parmi lesquelles le fait que Blanche n’avait pas aimé ce poème :

Je me rappelle que Blanche m'a dit que parmi mes poèmes «L'Escalier bleu » est le meilleur et que lorsque sa forme sera mise au point ce sera un beau poème. [...] / Par contre, elle n’a pas aimé «La Calèche » que j’aime tant: «Vous auriez peut-être dû l'écrire en prose quoiqu'il y ait là des notations de matières très justes ». (GM, p. 25.)

La parole de la Sibylle aurait-elle abouti longtemps après à cette autocensure de l'édition de 2009? Le poème est-il finalement apparu comme trop « sibyllin » au regard d'une poétique qui s'est simplifiée au fil du temps, et trop marqué par les signes du désir et de la sensualité érotique? La suppression est-elle une forme d'hommage secret au jugement de celle qui a tant compté dans la vie de Bauchau?

Un autre poème a survécu aux coupes sombres du poète, mais considérablement réduit en longueur et rendu très allusif dans ses images, il n'est éclairé que par son titre qui laisse imaginer l'anecdote, à

\footnotetext{
${ }^{40} \mathrm{D}$, p. 100.

${ }^{41}$ François Laruelle, Théorie générale des victimes, Paris, Mille et une nuits, 2012, p. 117.
} 
savoir «La volière ouverte ». Les manuscrits donnent à lire un poème initial de plus grande ampleur, évoquant d'abord le marché aux oiseaux où le père «en ses heures d'aveuglement» (A228) achetait parfois des «oiseaux prisonniers ». Le poème manuscrit raconte alors :

Olivier un matin a pris [...]

le bouvreuil et l'a lâché par la fenêtre.

Glissant ma main par la porte ouverte de la cage

j’ai pris le chardonneret

Avec sa jolie tête rouge et

noire, un peu de jaune

disant l'angoisse et je sentais

contre ma paume

tout l'évangile ${ }^{42}$ de son cœur

Gabrielle la gouvernante

l'archange détesté du bien

est entrée en tenant dans la main une règle

dont elle nous frappait méchamment

sur la tête

quand les parents n'étaient pas là

Elle nous regardait triomphante $[\ldots]$

Remettez cet oiseau. Je le dirai

à votre père

Je n'osais plus lâcher l'oiseat [...]

et déjà je sentais déjà les

dégoûtantes larmes

de la faiblesse désarmée

Mais Olivier baissant sa tête de bélier

Sortait le grand couteau un jour trouvé en terre

Le couteau du soldat avec son manche noir et sa lame ébréchée

Elle fuyait disant : je le dirai à votre père (A231 et A232)

Le souvenir a été entièrement édulcoré du poème final, très mince :

Par la grâce d'un front buté

ce jour ne fut pas oublié

Je sens toujours

la liberté dans ma main droite

\footnotetext{
${ }^{42}$ Mot de lecture douteuse, FHB A231.
} 
et le chant venir se poser

sur le fer de lame ébréché

du frère avec son couteau noir

«La volière ouverte », sans doute écrit parmi les derniers poèmes à la fin de l'année 1963, et l'on sent ici la recherche d'une langue plus nerveuse, plus tendue. Une hypothèse possible de cette réduction à un noyau allusif est qu'il est peut-être marqué par la relecture de Rimbaud pratiqué précisément en novembre 1963 :

Pour le moment j'éprouve un peu de fatigue envers les poèmes de la série La Fenêtre d'images, que j’ai tant aimés en les écrivant.

La relecture de Rimbaud m'est utile. Surtout par ce qu'il a de maigre et de musclé, le rejet de l'ornement et des rythmes tout faits. (D, p. 309 .)

L'approche des manuscrits de L'Escalier bleu est donc extrêmement féconde, notamment parce qu'on peut en coordonner l'étude avec les témoignages du poète dans son Journal de La Déchirure et avec le roman La Déchirure. Il faudrait y ajouter pour bien faire les manuscrits du roman et reconstituer le dossier génétique complet des deux textes écrits quasi simultanément pour affiner notre approche des modalités de la création chez Henry Bauchau. À ce stade cependant, ils nous paraissent livrer un certain nombre d'enseignements. Ces manuscrits apparaissent comme un lieu de libre cours avant d'être celui de la censure et de l'évaluation de soi. Le poète nous semble y confier sans frein sa difficulté d'être plus encore que dans les journaux ou récits biographiques, où elle est déjà recomposée dans une tentative de leçon. On y touche du doigt la manière dont, au-delà de l'expression de la mémoire et de la nostalgie du temps retrouvé, l'écriture vient exercer et apporter sa force sur le matériau même de la fragilité. L'élimination du matériau faible travaille comme une sangle qui va donner de la tenue au texte, et qui va, autant que possible, ériger l'auteur en sujet de son histoire. Et ce travail s'est poursuivi jusqu'au dernier souffle comme le montre l'édition ultime de 2009. Les manuscrits montrent aussi la résonance qu'il peut exister entre les lectures poétiques et les observations que fait le poète sur l'écriture d'autres poètes, l'imitation de tons et de formes à laquelle il se livre, à notre avis consciemment, avant là aussi de (re)prendre la maîtrise de sa 
propre voix en gommant ce que son écriture peut avoir d'emprunté. L'advenue de la forme est en même temps advenue de soi et cette approche des manuscrits montre de manière peut-être plus fine que l'œuvre réalisée combien l'écrivain construit son être par l'écriture.

Catherine MAYAUX Université de Cergy-Pontoise 